\title{
Asociación de hábitos alimentarios y estado nutricional con el nivel socioeconómico en adultos mayores que asisten a un Programa Municipal
}

\author{
Association of dietary habits and nutritional status with socioeconomic status of older \\ adults attending a Municipal Program
}

\author{
Fiorella Sánchez-Ruiz¹, Flor De la Cruz-Mendoza', María Cereceda-Bujaico', \\ Sissy Espinoza-Bernardo \\ Escuela Académico Profesional de Nutrición, Facultad de Medicina, UNMSM. Lima, Perú.
}

\begin{abstract}
Resumen
Introducción: La población de adultos mayores se viene incrementando en el país y su problemática es compleja. Es un grupo de riesgo, con problemas de salud y nutrición causados por una serie de factores, entre ellos los socioeconómicos. Objetivos: Determinar la asociación de los hábitos alimentarios y estado nutricional con el nivel socioeconómico (NSE) de los adultos mayores que asisten a un Programa Municipal. Diseño: Estudio descriptivo, transversal. Lugar: Programa Municipal del Adulto Mayor en cuatro distritos de Lima Metropolitana. Participantes: Personas mayores de 60 años de ambos sexos. Intervenciones: Se determinó el peso y la talla (por altura de rodilla) a 115 personas mayores de 60 años que asistían constantemente al Programa Municipal. Se les aplicó una encuesta sobre hábitos alimentarios y otra para establecer el nivel socioeconómico, ambas previamente validadas. Principales medidas de resultados: Estado nutricional según índice de masa corporal, hábitos alimentarios adecuados según recomendaciones y nivel socioeconómico según categorías de la Escala de Graffar. Resultados: Se encontró una prevalencia de sobrepeso $(42,6 \%)$ y obesidad (19,1\%). Más de $60 \%$ presentó hábitos alimentarios inadecuados respecto al consumo de carnes $(65,2 \%)$, lácteos $(78,3 \%)$, menestras $(87,8 \%)$, frutas y verduras $(64,3 \%)$. Los adultos mayores se ubicaron principalmente en el NSE medio bajo $(37 \%)$ y en el NSE medio medio (33\%). El estado nutricional no tuvo una asociación estadisticamente significativa con el NSE ( $p=0,629)$, mientras que para los hábitos alimentarios, se observó asociación con el NSE respecto al consumo de carnes $(p=0,003)$, cereales y tubérculos $(p=0,038)$ y de frutas y verduras $(p=0,001)$. Conclusiones: En nuestros sujetos de estudio se evidenció asociación entre los hábitos alimentarios con el NSE, pero no así para con el estado nutricional.
\end{abstract}

Palabras clave: Adulto mayor, estado nutricional, hábitos alimentarios, nivel socioeconómico, Municipalidad.

\section{Abstract}

Background: The population of older adults has been increasing in the country. Addressing their problems is complex. They are considered a risk group, with health and nutrition problems related to socioeconomic and other factors. Objectives: To determine the association of dietary habits and nutritional status with socioeconomic status (SES) in older adults attending a Municipal Program. Design: Descriptive, cross-sectional association study. Setting: Municipal Program for Older Adults of 4 districts in Metropolitan Lima, Peru. Participants: Adults of both sexes 60 years or over. Interventions: Weight and height were determined, and previously validated surveys on dietary habits and socioeconomic status were applied to 115 subjects who constantly attended a Municipal Program. Main outcome measures: Nutritional status according to body mass index, adequacy of eating habits according to recommendations, and socioeconomic status by Graffar's scale. Results: Overweight and obesity prevalence were respectively $42.6 \%$ and $19.1 \%$. Over $60 \%$ had inadequate dietary habits regarding meat $(65.2 \%)$, dairy $(78.3 \%)$, beans $(87.8 \%)$, fruits and vegetables $(64.3 \%)$. Older adults were located mainly on the medium-low SES (37\%) and medium-medium SES (33\%). Nutritional status was not significantly associated with SES $(p=0.629)$. Dietary habits were significantly associated $(P<0.05)$ with SES in the consumption of meat $(p=0003)$, cereals and tubers ( $p=0038$ ), and fruits and vegetables ( $p=0001)$. Conclusions: Association of food habits with SES but not with nutritional status was found in our study subjects.

Keywords: Older adults, nutritional status, dietary habits, socioeconomic status, Municipality.

An Fac med. 2014;75(2):107-11 / doi: http://dx.doi.org/10.15381/anales.v75i2.8382

\section{INTRODUCCIÓN}

La estructura demográfica global está cambiando debido a una marcada disminución en las tasas de fertilidad y al incremento en la esperanza de vida, originando un incremento en la población adulta mayor de los países latinoa- mericanos ${ }^{(1)}$. Cabe mencionar que, en América Latina, la esperanza de vida al nacer de los países de mayores ingresos supera en diez años a la de los países de menores ingresos ${ }^{(2)}$.

Actualmente, la población adulto mayor de 60 años ${ }^{(3)}$ representa el 9,1\% de la población total del Perú (4) y 33,8\% de esta población habita en Lima, representando un tercio de la población anciana del Perú (4-6). En Lima Metropolitana se observó un incremento en la población adulta mayor, pasando de 10\% de la población total en 2004 a $12,1 \%$ para $2010{ }^{(6)}$. 
El abordaje de la problemática de la población adulta mayor en Lima es complejo ${ }^{(7)}$, debido a que es considerada como grupo de riesgo por tener problemas de salud y nutrición causados por una serie de factores tanto físicos como sociales, fisiológicos y socioeconómicos ${ }^{(8)}$. Es por ello que el Ministerio de la Mujer y Desarrollo Social estipula la necesidad de realizar estudios que identifiquen los problemas reales de este grupo poblacional ${ }^{(9)}$.

Estudios realizados en Brasil y Chile concluyeron que los adultos mayores con menores ingresos familiares poseían factores de riesgo en cuanto a su estilo de vida, como el menor consumo de vegetales frescos, disminución en el consumo de lácteos y ejercicios menos frecuentes, así como una mayor incapacidad funcional ${ }^{(10,11)}$. Otro estudio en México concluyó que, para establecer el riesgo de desnutrición en el adulto mayor, es importante evaluar la situación económica y social, además de la información general de salud y las medidas antropométricas ${ }^{(12)}$.

En nuestro país, el Ministerio de Trabajo informó que 30,4\% de los adultos mayores es pobre ${ }^{(13)}$. Por otro lado, la empresa encuestadora Ipsos Apoyo, en su estudio de Perfil del Adulto Mayor 2010, indicó que la mayor parte de los ingresos del adulto mayor proviene del apoyo de sus hijos y de su jubilación ${ }^{(14)}$. Asimismo, el 60\% de la PEA adulta mayor de Lima Metropolitana no tiene acceso a pensión de jubilación ${ }^{(15)}$, siendo esto un $70 \%$ a nivel nacional ${ }^{(13)}$.

La alimentación tiene un papel muy importante en el proceso del envejecimiento ${ }^{(16)}$. El estilo de vida de los adultos mayores unido a los malos hábitos alimentarios, pobreza, la poca disponibilidad de recursos, el aislamiento enfermedades crónicas y degenerativas comprometen el consumo de alimentos y por ende su estado nutricional, afectando negativamente la funcionalidad del adulto mayor y reduciendo su calidad de vida ${ }^{(17)}$.

Un estudio realizado en Cuba, en el año 2007, observó que aproximadamen- te la mitad de la población presentaba obesidad ${ }^{(18)}$, y otro estudio encontró que $50 \%$ de la mujeres adultas mayores era obesa ${ }^{(19)}$. En el Perú, un estudio halló que conforme incrementaba la edad, la prevalencia de desnutrición aumentaba y la obesidad disminuía (20). Y en otro estudio, 36,2\% de las mujeres y $18,1 \%$ de los hombres presentaron sobrepeso ${ }^{(21)}$. Por otro lado, en la población adulta mayor existe una relación directa entre hábitos alimentarios inadecuados y enfermedades crónicas no transmisibles ${ }^{(22)}$.

Un estudio realizado en Lima Metropolitana, en el año 2006, analizó el efecto de las condiciones socioeconómicas en el estado nutricional del adulto mayor, hallando que la prevalencia de obesidad fue $22 \%$ y tuvo una diferencia significativa respecto al nivel socioeconómico ${ }^{(23)}$.

Según el Instituto de Gerontología de nuestro país, son pocos los estudios realizados en este grupo, y de ellos, la mayoría se realizó en adultos mayores institucionalizados u hospitalizados ${ }^{(24)}$.

Es por ello que el presente estudio tuvo como objetivo determinar la asociación de los hábitos alimentarios, estado nutricional con el nivel socioeconómico de los adultos mayores que asisten constantemente al Programa Municipal de Lima Metropolitana.

\section{MÉTODOS}

Estudio descriptivo, transversal, de asociación cruzada. La muestra estuvo constituida por 115 adultos mayores de 60 años de ambos sexos, orientados en tiempo y espacio, y con capacidad de valerse por sí mismos, que asistían constantemente al Programa Municipal del Adulto Mayor, con ubicación en los distritos del Agustino, Cercado de Lima, Los Olivos y San Juan de Miraflores. El estudio se realizó entre los meses de enero y abril del año 2011. El muestreo fue no probabilístico por conveniencia. Previo al estudio se solicitó el consentimiento informado a los participantes.
Para evaluar el estado nutricional se tomó las medidas de peso -para lo cual se utilizó una balanza de pie- y la talla, que midió la altura de rodilla con un estadiómetro de metal, para estimar la talla según la fórmula de Bermúdez, dado que él hace la adaptación para sujetos hispano americanos ${ }^{(25-26)}$ :

Hombres: $69,11+(1,86 * A R)-(0,03 * E)$

Mujeres: $72,08+(1,84 * A R)-(0,131 * E)$

Donde: $A R=$ altura de rodilla, $E=$ edad.

Las mediciones fueron realizadas según la Norma Técnica para la Valoración Nutricional Antropométrica del Adulto Mayor elaborada por el CENAN/INS ${ }^{(27)}$.

Para determinar los hábitos alimentarios se utilizó una encuesta de frecuencia de consumo diario y semanal de los grupos de alimentos por porciones recomendadas, así como preguntas sobre consumo de bebidas e inhibidores, adición de sal a las preparaciones y tiempos de comida ${ }^{(28)}$. Este instrumento fue sometido a evaluación por un grupo de jueces expertos.

Para determinar el nivel socioeconómico se aplicó una encuesta basada en la escala de Graffar modificada por Méndez-Castellano (29), la cual está dividida en cuatro partes: el nivel de instrucción y actividad laboral del jefe del hogar; el grado de instrucción de la esposa del jefe del hogar y la última parte está dirigida a las características de la vivienda, material predominante de esta y saneamiento con los que cuenta; cada una con su respectivo puntaje para clasificar a la población según su nivel socioeconómico.

Los datos fueron procesados en una hoja de cálculo de Microsoft Excel 2007. El estado nutricional se determinó según el índice de masa corporal (IMC), utilizando los puntos de corte para el adulto mayor referidos por el CENAN-INS -MINSA ${ }^{(27,30)}$ : peso bajo IMC $\leq 23$, normal $\mathrm{IMC}>23$ a $<28$, sobrepeso IMC $\geq 28$ a $<32$ y obesidad IMC $\geq 32$. Se consideró los hábitos alimentarios como: 'adecuados' (consumo de porciones de alimentos según lo re- 
comendado) e 'inadecuados' (consumo de alimentos por debajo o encima de las porciones recomendadas). El nivel socioeconómico fue determinado mediante la escala de Graffar obteniéndose las categorías de nivel socioeconómico: alto, medio alto, medio medio, medio bajo y bajo.

Para el análisis de la información se utilizó el paquete estadístico SPSS 18.0, realizando un análisis descriptivo con medidas de tendencia central y análisis inferencial para establecer la asociación de las variables. Se determinó la asociación de cada uno de los indicadores de la variable hábitos alimentarios y estado nutricional con la variable nivel socioeconómico aplicando la prueba $X^{2}$, con un nivel de confianza de $95 \%$ (se asumió un error de $5 \%$, es decir, una $\mathrm{p}<0,05)$.

\section{RESULTADOS}

La muestra estuvo constituida por 115 adultos mayores, de los cuales $97 \%$ fueron mujeres y $2 \%$ varones, con una edad promedio de $71 \pm 7,6$ años y rango de 60 a 93 años. El 70\% de la muestra estuvo comprendida en el rango de 60 a 74 años.

La mayoría de la población se ubicó en el nivel socioeconómico (NSE) medio medio y medio bajo, según la Escala de Graffar modificada ${ }^{(29)}$, encontrándose un poco más del tercio de la población en el NSE medio medio y otro tercio en el medio bajo. Por otro lado, solo una persona se ubicó en el NSE alto, representando $0,9 \%$ de la población.

Con respecto al estado nutricional de la población estudiada, se obtuvo una media del IMC de $29 \pm 3,8$ con un IMC máximo de 40,7. Así mismo, 19,1\% de la población presentó obesidad, $42,6 \%$ sobrepeso, $29,6 \%$ peso normal y $8,7 \%$ peso bajo. Se pudo apreciar que conforme la edad avanzaba la prevalencia de obesidad disminuía.

Por otro lado, se observó que más de $60 \%$ de la población adulta mayor presentó hábitos inadecuados para el

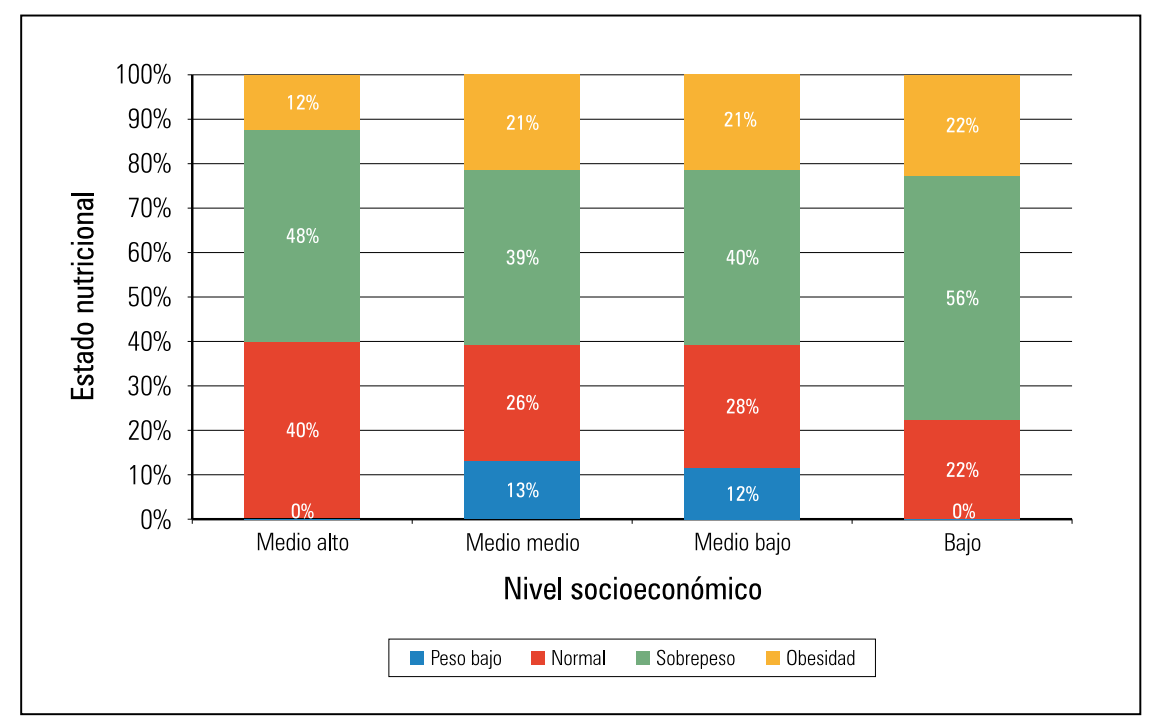

Figura 1. Estado nutricional según nivel socioeconómico de los adultos mayores.

consumo de carnes $(65,2 \%)$, lácteos $(78,3 \%)$, menestras $(87,8 \%)$ y de frutas y verduras $(64,3 \%)$.

$\mathrm{Al}$ asociar el estado nutricional con el NSE (figura 1), se observó que el sobrepeso y obesidad estuvo presente en todas las categorías del NSE, con porcentajes casi similares excepto para el NSE bajo, donde la prevalencia de sobrepeso $(56 \%)$ fue mayor. Solo se detectó peso bajo en el NSE medio bajo y NSE medio medio. A pesar de ello, no se encontró asociación significativa entre las variables estado nutricional y NSE $(p=0,629)$. En relación a la obesidad, esta fue ligeramente mayor en los del NSE bajo (22\%).

$\mathrm{Al}$ asociar hábitos alimentarios con NSE, se observó una asociación significativa $(p<0,05)$ con la variable NSE en solo tres de los doce indicadores de la variable hábitos alimentarios, que corresponden al consumo de alimentos, siendo estos el consumo de carnes $(p=0,003)$, frutas y verduras $(p=0,001)$, cereales y tubérculos $(p=0,038)$. Se evidencia que el grupo de adultos mayores del NSE medio alto tuvo mayor porcentaje de hábitos adecuados en comparación con los otros NSE (tabla 1).

Se observó que los adultos mayores que pertenecían al NSE medio bajo y
NSE bajo tuvieron 3 veces más probabilidad de tener un hábito inadecuado de consumo de carnes $(O R=3,18)$; de igual modo, respecto al consumo de frutas y verduras, hubo 5 veces más probabilidad de tener un hábito inadecuado en su consumo $(O R=4,93)$.

\section{DISCUSIÓN}

Es evidente que las mayores posibilidades para la supervivencia, y el cuidado de la salud la tienen los ancianos del estrato social alto o medio alto con mayor poder económico. En los sectores populares, en cambio, la pobreza margina a los mayores de una atención de primera categoría.

Desde la mitad del siglo XX en adelante, la malnutrición en los ancianos ha sido un problema importante de salud pública, ya que aumenta la morbimortalidad ${ }^{(30)}$.

La alta prevalencia de sobrepeso y obesidad encontrada en nuestro estudio es similar a lo hallado en un estudio realizado en el adulto mayor del CAMLima Cercado, que encontró una prevalencia de $47 \%$ de sobrepeso y $19 \%$ de obesidad ${ }^{(31)}$. Asimismo, otro estudio realizado en un distrito aledaño a Lima Metropolitana halló una prevalencia de 
Tabla 1. Hábitos alimentarios y su asociación con el nivel socioeconómico de los adultos mayores.

\begin{tabular}{|c|c|c|c|c|c|c|c|c|c|c|}
\hline \multirow{3}{*}{ Hábitos alimentarios } & & \multicolumn{8}{|c|}{ Nivel socioeconómico } & \multirow{3}{*}{$P$} \\
\hline & & \multicolumn{2}{|c|}{$\begin{array}{c}\text { Medio alto } \\
n=25\end{array}$} & \multicolumn{2}{|c|}{$\begin{array}{c}\text { Medio medio } \\
\mathrm{n}=38\end{array}$} & \multicolumn{2}{|c|}{$\begin{array}{c}\text { Medio bajo } \\
n=43\end{array}$} & \multicolumn{2}{|c|}{$\begin{array}{l}\text { Bajo } \\
n=9\end{array}$} & \\
\hline & & $\mathrm{N}^{0}$ & $\%$ & $\mathrm{~N}^{0}$ & $\%$ & $\mathrm{~N}^{0}$ & $\%$ & $\mathrm{~N}^{0}$ & $\%$ & \\
\hline \multirow{8}{*}{$\begin{array}{l}\text { Consumo de alimentos } \\
\text { por porciones } \\
\text { recomendadas }\end{array}$} & Carne & 16 & 64 & 13 & 34 & 10 & 23 & 1 & 11 & 0,003 \\
\hline & Lácteos & 7 & 28 & 10 & 26 & 6 & 14 & 2 & 22 & 0,462 \\
\hline & Menestras & 3 & 12 & 3 & 8 & 8 & 19 & 0 & 0 & 0,313 \\
\hline & Frutas y verduras & 16 & 64 & 16 & 42 & 8 & 19 & 1 & 11 & 0,001 \\
\hline & Cereales y tubérculos & 11 & 44 & 23 & 61 & 25 & 58 & 1 & 11 & 0,038 \\
\hline & Aceite de oliva & 16 & 64 & 17 & 45 & 17 & 40 & 2 & 22 & 0,109 \\
\hline & Agua & 3 & 12 & 2 & 5 & 3 & 7 & 1 & 11 & 0,770 \\
\hline & Frituras y/o snacks & 5 & 20 & 7 & 18 & 8 & 19 & 1 & 11 & 0,948 \\
\hline \multicolumn{2}{|l|}{ Tiempos de comida* } & 10 & 40 & 8 & 21 & 9 & 21 & 1 & 11 & 0,197 \\
\hline \multicolumn{2}{|c|}{ Consumo de inhibidores ${ }^{* *}$} & 11 & 44 & 21 & 55 & 19 & 44 & 2 & 22 & 0,326 \\
\hline \multicolumn{2}{|c|}{ Adición de sal a sus preparaciones servidas ${ }^{* * *}$} & 17 & 68 & 30 & 79 & 32 & 74 & 8 & 89 & 0,591 \\
\hline
\end{tabular}

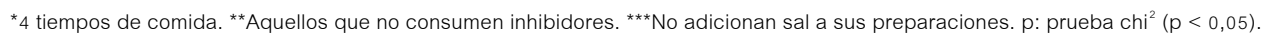

sobrepeso de $55 \%$ y $8 \%$ de obesidad ${ }^{(32)}$. En otros países, como en Ecuador, Brasil, Chile y Cuba, también se encontró prevalencias altas de sobrepeso y obesidad en este grupo ${ }^{(16,18,33,34)}$.

El estado nutricional por cada NSE en el grupo estudiado coincide con lo hallado en un estudio realizado en Chile ${ }^{(11)}$, que comparó dos grupos de adultos mayores, uno de ingreso familiar alto y otro bajo, encontrando sobrepeso en ambos grupos, con una mayor prevalencia (73\%) en el de ingreso bajo, lo cual también se halló en nuestro estudio, presentando el grupo del NSE bajo un $56 \%$ de sobrepeso.

A pesar de ello, no se halló una asociación significativa del estado nutricional en relación al NSE. Esto podría deberse a que la proporción de sobrepeso y obesidad fue similar en todos los NSE. Si bien todos los NSE presentaron sobrepeso y obesidad, su origen podría no ser el mismo. Según Peña y Bacallao ${ }^{(35)}$, sus causas podrían ser distintas, ya que en los niveles de mayores ingresos las causas de la obesidad están relacionadas al consumo de alimentos industrializados altos en grasas y carbohidratos como por ejemplo golosinas, snacks, comidas rápidas o pre-elaboradas, además de un aumento en el tamaño de las porciones y disminución de la actividad física, y un mayor sedentarismo que implica los trabajos en la vida moderna o jubilación, mientras que los obesos de los NSE bajos pueden presentar un incremento en su peso corporal causado por un excesivo consumo de productos de panadería, fideos, arroz, azúcares simples, alcohol, gaseosas, entre otros.

En forma general, los hábitos alimentarios en nuestra población de estudio fue inadecuado, debido a que en su mayoría no consumían las porciones de alimentos recomendadas según las Guías de Alimentación para el Adulto Mayor ${ }^{(28)}$, como es el bajo consumo de lácteos que también se observa en otros países, como en Chile, en donde se encontró que el $25 \%$ de su población consumía 2 porciones de lácteos al día ${ }^{(36)}$. De lo observado, es probable que este grupo no esté alcanzando el nivel de ingesta de calcio con la dieta.

Por otro lado, el consumo de agua hallado en este grupo es preocupante, debido a que un consumo bajo lleva al riesgo de deshidratación y, si se produce de una manera crónica, modifica la capacidad del organismo para mantener la homeostasis en cualquier situación fuera de lo normal, pudiendo afectar la salud del individuo.

De manera similar, otro estudio realizado en Chile con dos grupos de adultos mayores de niveles educativos distintos, halló que la alimentación era inadecuada en cantidad y calidad respecto al grupo de nivel educativo bajo, y su frecuencia de consumo estaba por debajo de las recomendaciones del Ministerio de Salud de Chile, especialmente respecto al consumo de lácteos y de agua en ambos grupos ${ }^{(16)}$.

En nuestro estudio encontramos asociación $(p<0,05)$ entre el NSE con el consumo de carnes, consumo de cereales y tubérculos y consumo de frutas y verduras. El grupo SE medio alto presentó un mayor consumo de porciones recomendadas, frente al grupo del NSE bajo.

El consumo bajo de frutas y verduras evidenciado en el grupo de NSE bajo fue similar a lo informado en otro estudio realizado en Brasil, que demostró asociación con el NSE, ya que familias de ingreso bajo presentaron menor consumo de frutas y verduras respecto a las de ingresos altos ${ }^{(10)}$. Según Peña ${ }^{(31)}$, esto podría atribuirse al elevado precio principalmente de las frutas, que las hacen inaccesibles para los grupos de ingresos más bajos. Por otro lado, este consumo bajo estaría implicando un ingesta baja de fibra en esta población, y por ende el incremento de riesgo para las dislipidemias, diabetes, cáncer al 
colon, entre otras enfermedades de alta prevalencia en la edad avanzada ${ }^{(13,35)}$.

Aunque no se encontró asociación entre el NSE y el consumo de menestras, el NSE bajo presentó un menor consumo de estas. Por otro lado, este grupo presentó un elevado consumo de frituras y/o snacks, siendo favorecida por su fácil obtención, en mercados, quioscos y restaurantes de comida rápida ${ }^{(36)}$.

Los hábitos alimentarios inadecuados detectados en más de $60 \%$ de los adultos mayores pueden aumentar el riesgo de deficiencias nutricionales y afectar su calidad de vida.

\section{REFERENCIAS BIBLIOGRÁFICAS}

1. Novelo LH. Situación epidemiológica y demográfica del adulto mayor en la última década. Rev Salud Pub Nutr Méx 2003;5.

2. Pichihua J, Miranda M, Sánchez J, Trujillo $H$, Salvatierra R. Efecto del nivel socioeconómico sobre algunos indicadores de salud y nutrición en la ni-ez, Perú 2003-2004. Lima: Instituto Nacional de Salud; 2007.

3. Inga J, Vera A. Calidad y satisfacción de vida de adultos mayores de 60 a-os en Lima-Perú. Estudio piloto y análisis psicométrico. [Monografía en Internet] Asociación por la Defensa de las Minorías. [Fecha de acceso 3 de Julio de 2011]; 12(5). URL disponible en: http//www.geocities.com/Heartland/ Cabin/5900/adulto.htm.

4. Instituto Nacional de Estadistica e Informática. Censos Nacionales 2007: XI de Población y VI de Vivienda: Perfil Sociodemográfico del Perú. 2da Edición de 2008. Perú: INEI; 2008.

5. Foro de la Sociedad Civil en Salud, Mesa Temática de Adultos Mayores. Vigilancia ciudadana a los servicios de salud desde la percepción de los adultos mayores en dos distritos de Lima Metropolitana. Perú: Foro de la Sociedad Civil en Salud; 2008.Foro de la Sociedad Civil en Salud, Mesa Temática de Adultos Mayores. Vigilancia ciudadana a los servicios de salud desde la percepción de los adultos mayores en dos distritos de Lima Metropolitana. Perú: Foro de la Sociedad Civil en Salud; 2008.

6. Instituto Nacional de Estadística e Informática. Situación de la Niñez y del Adulto Mayor. Perú: INEl; 2011. (Informe Técnico Nº2 Junio 2011).

7. Ministerio de Salud, Ministerio de la Mujer y Desarrollo Social, Red de Municipios y Comunidades Saludables, Organización Panamericana de la Salud. Politicas saludables para adultos mayores en los municipios. Lima: OPS; 2004. (Cuadernos de Promoción de la Salud N${ }^{\circ} 12$ ).

8. Castro V, Sánchez NJ, Tapia CR. Las enfermedades crónicas en las personas de 60-69 a-os. Salud Pública Mex. 2006;38:438-447.

9. Ministerio de la Mujer y Desarrollo Social. Plan Nacional para las personas Adultas Mayores 2006 - 2010. Perú: MINDES; 2006.

10. Lima-Costa MF, Barreto SM, Firmo J, Uchoa E. Socioeconomic position and health in a popula- tion of brazilian elderly: the Bambui Health and Aging Study (BHAS). Pan Am J Public Health. 2003;13(6):387-93. doi: http://dx.doi.org/10.1590/ S1020-49892003000500007

11. Labra-a T, Durán F, Asenjo I. Nivel de ingreso familiar y su relación con la calidad de la dieta de 2 grupos de adultos mayores de 60 a-os de la ciudad de Concepción Chile. Rev Chil Nutr. 1999;26(3):301-9.

12. Franco N, Ávila J, Ruiz L, Gutiérrez L. Determinantes del riesgo de desnutrición en los adultos mayores de la comunidad: Análisis secundario del estudio Salud, Bienestar y Envejecimiento (SABE) en México. Rev Panam Salud Pública. 2007;22(6):369-75

13. Ministerio de Trabajo y Promoción del Empleo. Informe anual del empleo de las personas adultas mayores - 2007. Lima: MINTRA; 2008.

14. Ipsos APOYO Opinión y Mercado. Perfil del adulto mayor 2010, [sitio en Internet] 2010. [fecha de acceso 15 de Junio de 2011]; 10(177). URL disponible en: http://www.ipsos-apoyo.com.pe/sites/default/ files/marketing_data/MKT\%20Perfil\%20del\%20 adulto\%20mayor\%202010.pdf

15. Varela L, Chávez H, Herrera A, Méndez F, Gálvez M. Perfil del adulto mayor Perú - INTRA II. Desarrollando respuestas integradas de los sistemas de cuidados de la salud para una población en rápido envejecimiento. Lima: Instituto de Gerontologia/OMS; 2004.

16. Barrón MV, Mardones MA, Vera S, Ojeda G, Rodríguez F. Comparación de estilos de vida de la población económicamente activa mayor de 65 a-os entre las ciudades de Chillan y Valparaiso, Chile. Rev Theoria. 2006;15(1):33-44.

17. Reyes V, Sotomayor S, González L. Conductas relacionadas con la salud del adulto mayor en una comunidad suburbana del D.F. Rev Enferm Inst Mex Seguro Soc. 2007;15(1):27-31.

18. Abreu C, Viamontes A, Capote A, Betancourt M Estado nutricional en adultos mayores: casa de abuelos Amalia Simoni. AMC [revista en Internet] 2009 Agosto. [Fecha de acceso 10 de Junio de 2011]; 13(4). URL disponible en: http://scielo. sld.cu/scielo.php?script=sci_arttext\&pid=S102502552009000400008\&lng=es.

19. Abreu C, Burgos y, Ca-izares D, Viamontes A. Estado nutricional en adultos mayores. AMC [revista en internet] 2007 Octubre. [Fecha de acceso 10 de Junio de 2011]; 12(5). URL disponible en: http://scielo. sld.cu/scielo.php?script=sci_arttext\&pid=S102502552008000500005\&lng=es.

20. Sandoval L, Varela L. Evaluación del estado nutricional de pacientes adultos mayores ambulatorios. Rev Med Hered. 1998;9(3):104-8.

21. Cereceda M. Antropometria nutricional de adultos mayores. CAM Fiori Lima-Perú. 2003. An Fac med. UNMSM 2004;64(3 Supl II):31.

22. Ludeña A. Torres C, Bernui I. Antecedentes alimentarios del adulto mayor con y sin hipertensión primaria actual, en el hospital geriátrico San José PNP. San Miguel. Abril 2004. An Fac med. 2004;65(3 Supl II):44

23. Cárdenas $\mathrm{H}$. Efecto de las condiciones socio económicas en el estado nutricional del adulto mayor de Lima Metropolitana [Tesis doctoral]. Lima: Universidad Nacional Federico Villarreal; 2007

24. Varela L, Ortiz P, Chávez H. Características de los trabajos científicos nacionales en geriatría y gerontología 1980-2001. Rev Med Hered. 2003;14:18-25.

25. Bermúdez O, Tucker K. Uso de la altura de rodilla para corregir la talla de ancianos de origen hispa- no. Rev ALAN. 2000;50(1):42-7.

26. Acosta R. Evaluación nutricional en ancianos. Córdoba: Editorial Brujas; 2008.

27. Instituto Nacional de Salud, Centro Nacional de Alimentación y Nutrición. Valoración nutricional antropométrica del adulto mayor. Lima: INS/ CENAN; 2005

28. Ministerio de la Mujer y Desarrollo Social, Dirección de Personas Adultas Mayores. Guia Técnica Alimentaria para Personas Adultas Mayores. Lima: MINDES; 2009.

29. Feijoo F. Evaluación de la calidad de vida en el adulto mayor y su relación con el estado nutricional en Lima Metropolitana. [Tesis Doctoral].Lima: Unidad de Postgrado, Universidad Nacional Federico Villareal; 2008

30. Benjumea GL. Estado nutricional como determinante de la calidad de vida de los adultos mayores del programa de comedores de la zona sur oriental de Sincelejo, Sucre - Colombia; 2010.

31. Peña M, Bacallao J. La obesidad en la pobreza: Un problema emergente en las Américas. En: Peña M, Bacallao J, eds. La Obesidad en la Pobreza: Un Reto para la Salud Pública. Washington, DC: Organización Panamericana de la Salud; 2000:3-11.

32. Cárdenas H, Bello C, Feijóo M, Huallpa E. Evaluación nutricional de un grupo de adultos mayores residentes en Perú. Rev Cubana Salud Pública. 2004;30(3).

33. Carrera M, Jaramillo L, Consumo alimentario ideal, indice de masa corporal y salud bucal en adultos mayores organizados de la ciudad de Quito. Rev Med Vozades. 2009;20:23-6.

34. Cereceda P, Gonzales H, Carranza E, Zúñiga H, Cuellar E, Vásquez D, Indicadores antropométricos y su relación con enfermedades crónicas no transmisibles - CAM Lima Cercado. An Fac med. 2004;65(supl 2004):35.

35. Borba R, Coelho M, Borges P, Corrêa J, González C. Medidas de estimación de la estatura aplicadas al índice de masa corporal (IMC) en la evaluación del estado nutricional de adultos mayores. Rev Chil Nutr. 2008;35 (Supl 1):272-9.

36. Restrepo S, Morales R, Ramírez C, López M, Varela L. Los hábitos alimentarios en el adulto mayor y su relación con los procesos protectores y deteriorantes en salud. RCN [revista en Internet] 2006. [fecha de acceso 10 de Junio de 2011]; 33(3). URL disponible en: http://www.scielo. $\mathrm{cl} /$ scielo.php?script=sci_arttext\&pid=S071775182006000500006 .

Artículo recibido el 3 de setiembre de 2013 y aceptado para publicación el 29 de enero de 2014.

Financiamiento:

Trabajo con financiamiento propio de los autores.

Conflictos de intereses:

Los autores declaran no existir conflictos de interés.

\section{Correspondencia:}

Fiorella Xixjuddhe Sánchez Ruiz

Urb. Alfonso Cobian Mz F28, Chaclacayo

Teléfono: 971901586

Correo electrónico: fiore_nutrast@yahoo.com 